\title{
Curved-Folding-Inspired Deployable Compliant Rolling-contact Element (D-CORE)
}

Todd Nelson

Brigham Young University - Provo

Robert Lang

Spencer P. Magleby

Larry L. Howell

Brigham Young University - Utah, Ihowell@byu.edu

Follow this and additional works at: https://scholarsarchive.byu.edu/facpub

Part of the Mechanical Engineering Commons

\section{Original Publication Citation}

Nelson, T. G., Lang, R. J., Magleby, S. P., \& Howell, L. L. (2015). Curved-folding-inspired deployable compliant rolling-contact element (D-CORE). Mechanism and Machine Theory.

\section{BYU ScholarsArchive Citation}

Nelson, Todd; Lang, Robert; Magleby, Spencer P.; and Howell, Larry L., "Curved-Folding-Inspired Deployable Compliant Rolling-contact Element (D-CORE)" (2015). Faculty Publications. 1562.

https://scholarsarchive.byu.edu/facpub/1562 


\title{
Curved-Folding-Inspired Deployable Compliant Rolling-contact Element (D-CORE)
}

\author{
Nelson, Todd $\mathrm{G}^{a}$; Lang, Robert $\mathrm{J}^{b}$; Magleby, Spencer $\mathrm{P}^{a}$; Howell, Larry L ${ }^{a}$,* \\ ${ }^{a}$ Brigham Young University, Department of Mechanical Engineering, 435 CTB, Provo, UT \\ 84602, United States \\ ${ }^{b}$ Lang Origami, Alamo CA 94507, United States \\ * Corresponding author: Tel: +1 801 422 8037; Email: lhowell@byu.edu \\ Published article available with DOI 10.1016/j.mechmachtheory.2015.05.017
}

\begin{abstract}
This work describes a deployable compliant rolling-contact element joint (DCORE joint) that employs curved-folding origami techniques to enable transition from a flat to deployed state. These deployable joints can be manufactured from a single sheet of material. Two fundamental configurations of the D-CORE are presented. The first configuration allows for motion similar to that of a Jacob's ladder when the joint is in a planar state while achieving the motion of a CORE when in the deployed state. The second configuration constrains all degrees of freedom to create a static structure when the joint is in the planar state and allows for the motion of a CORE in the deployed state. Curved-folding origami techniques can be used with both configurations to control the cam radius and range of motion of the D-CORE. Physical models are demonstrated in Tyvek®, polycarbonate, polypropylene, and metallic glass. A deployable translating platform constructed of an inversion of the D-CORE is also shown.

Keywords: deployable, CORE, compliant mechanisms, origami, curved folding
\end{abstract}

\section{Introduction}

This work draws upon curved-folding techniques from origami to create a compliant rolling-contact element (CORE) [1] with the capability of being deployed from a planar state to a deployed state. CORE joints are largedisplacement angle, compliant, multi-stable, revolute joints [1]. CORE joints and other types of revolute joints have been investigated to mitigate the challenges encountered by traditional pin-joints such as backlash and wear. Wear can intensify undesired motion as the clearance between the pin and the hole increases with use. Traditional bearings present another alternative but can significantly increase cost, weight, and size. The CORE uses compliant flexures 


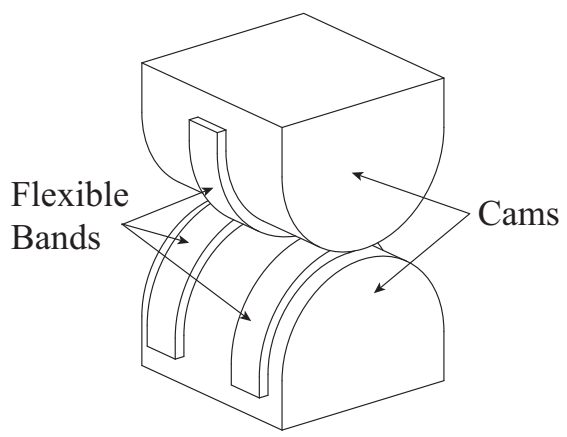

Figure 1: A CORE with circular cams.

that join two cams together, as shown in Figure 1. The tension in the flexures holds the cams together as they roll across each other.

While still preserving the desirable properties of CORE joints to reduce undesired motion and wear, deployable CORE (D-CORE) joints enable the joint to be stored in a compact position before and after use. This facilitates use of the joint in applications where space is a constraint before or after the joint is to function. This constraint is commonly seen in spacecraft, aircraft, minimally invasive surgery devices, and stowable furniture. The ability of the D-CORE to be manufactured from a single sheet of material and assembled through folding make it a low cost alternative to a traditional joint. The DCORE, as well as the CORE, differ from traditional pin joints as the axis of rotation moves during the rotation of the joints. While this prohibits the use of the D-CORE or CORE in some precision movement applications where a fixed axis of rotation is required, the movement of the axis of rotation is calculable enabling predicted motion paths. Another desirable property of one of the DCORE configurations discussed in this paper is the ability to be a zero-degreeof-freedom static structure in its stowed, planar state and to transition to a one-degree-of-freedom mechanism in its deployed state. This type of mechanism is known as a metamorphic mechanism [2].

\section{Background}

The use of compliant flexures in the D-CORE and CORE to join two rigid bodies together is reminiscent of a single unit in a Jacob's ladder toy. The DCORE is created using curved-fold origami techniques to construct the curved surfaces of the cams. This enables the joint to maintain the advantages of the CORE while adding the capability of the joint to be deployed to and from a flat position.

\subsection{Jacob's Ladder}

The underlying mechanism of the CORE is also what creates the optical illusion of a falling block in a Jacob's Ladder. The Jacob's Ladder is an ancient

Preprint: Published article available with DOI 


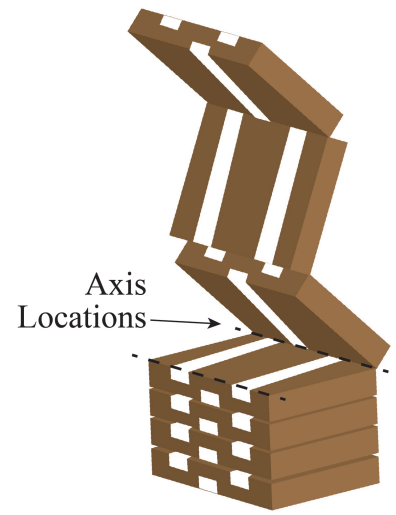

Figure 2: A Jacob's Ladder toy with hinge axis locations shown.

toy [3] that consists of ribbons connected in a specific pattern to wooden blocks, as shown in Figure 2. When the top block of a Jacob's Ladder is rotated, the illusion of a falling block is created as each block flips in alternating directions one after another. Similar to the study of the underlying mechanism of a color changing toy ball by Ding et al. [4], insights into mechanism design can be gained from an investigation of the Jacob's Ladder.

By isolating a single unit of the Jacob's ladder one can see how two rotational axes are present about which the top block can revolve, as shown in Figure 3. All of the degrees of freedom are constrained through either contact of the faces of the block and the ribbon or tension in the ribbon. Similarly, in the CORE all degrees of freedom besides the rolling motion are constrained through contact of the flexures and the cams or tension in the flexures. However, the introduction of a curved surface as the cam face rather than a flat surface as in the Jacob's Ladder causes the instantaneous axis of rotation to follow the curvature of the cam throughout the motion rather than switching between two stable axes of rotation. This difference in location of the instantaneous axis of rotations during the joint motions is shown in Figure 4.

\subsection{Compliant Mechanisms}

The CORE is a compliant mechanism, whose motion is derived at least partly from flexing members [5]. Compared to traditional mechanisms, compliant mechanisms can offer cost reduction due to lower part count and assembly time, simplified manufacturing processes, performance improvement through increased precision and reliability, and reduced wear, weight, and maintenance [5]. In the CORE, there is no need for lubrication, wear is dramatically reduced, and the compliance of the CORE enables design for specific force-deflection properties and an ease of miniaturization. 


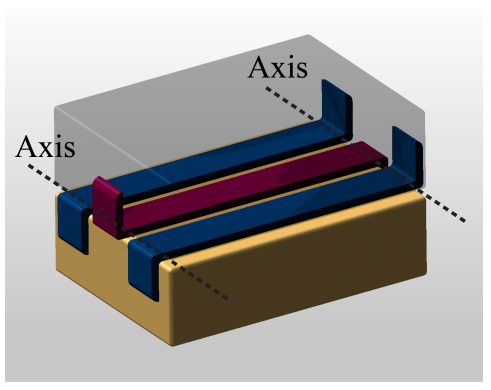

Figure 3: A single functional unit of a Jacob's Ladder in a closed position with hinge axes shown. The top block is transparent to show the attachment scheme of the bands.

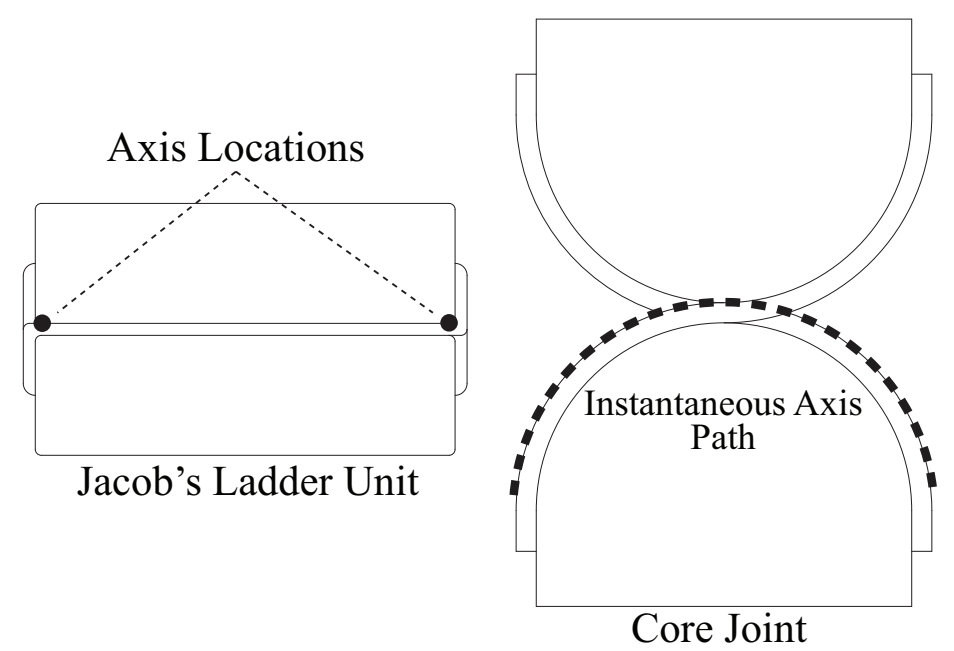

Figure 4: Location of the instantaneous axis of rotation of the Jacob's Ladder fundamental unit and a CORE through the joint motion. 


\subsection{Contact-Aided Rolling Joints}

The development of the CORE was preceded by similar mechanisms that utilize the mechanism described in the Jacob's Ladder. The Rolamite concept was developed in the 1960's [6]. This design used a band between two rollers to create linear displacement with little friction. Further development of the Rolamite has occurred as seen in the creation of analysis techniques of the joint [7], implementation of the concept in a prosthetic knee joint [8], and more recently using the concept to create variable joint stiffness [9].

The CORE [1] uses flexible bands to join two rolling surfaces similar to the Rolamite. However, while the Rolamite joint provides a linear displacement, the CORE allows the two rolling surfaces to roll over top of each other creating a motion similar to a revolute joint. Halverson et al. [1] used a strain energy approach to derive models for the CORE. Their work particularly examines tension-based multi-stable CORE joints and develops equations to describe the angular velocities of the cams, stress in the joint, strain of the flexures, and retaining forces (forces in the flexures) for these joints. A variation of this joint was used to create an artificial disc implant for the human spine designed to mimic the motion and stiffness characteristics of a human spinal disc [10].

The ability to scale the CORE and mechanisms similar to it has resulted in their use in medical devices for minimally invasive surgery [11] and robotic components such as a bio-mimetic finger [12].

\subsection{Origami Inspired Mechanisms}

Origami constructions have inspired the study a variety of mechanisms and folding structures [13] including planar-spherical over-constrained mechanisms [14] and queer-square mechanisms with multi-furcation qualities [15]. Folding techniques have been used to formulate a systematical approach to carton folding [16]. Most of these origami constructions used in mechanism analysis are prismatic, where the straight creases can be modeled as revolute joints and the panels as links as is the case in the development of a systematic approach to modeling carton folding [16]. Kirigami, which uses cutting as well as folding, has also inspired mechanism design [17].

Curved-fold origami is intrinsically tied to curved surfaces. When paper is folded along a curved crease, the two surfaces created are developable surfaces [18]. Developable surfaces are sometimes called singly-curved surfaces as at every point of the surface one of the principle curvatures is zero [19, 20]. They are a subset of ruled surfaces, or surfaces where a straight line, or ruling line, can be used to generate the surface [19]. Three types of developable surfaces exist: generalized cylinders, generalized cones, and tangent-developable surfaces [21]. Curved-fold origami can thus be thought of as a combination of developable surface patches that are joined through curved creases. David Huffman [22] [23] and Ron Reusch [24] pioneered a rigorous approach to curved folding with their work in the 1970's. Recent work by Lang [25] and Demaine et al. [26] has demonstrated methods to design curved-fold structures by treating the ruling lines of the developable surfaces as optics lines and curved-creases as lenses.

Preprint: Published article available with DOI 
Curved-folding techniques are used in this research to create the curved surfaces of the CORE. In addition, curved-folding enables the CORE joint to be stowed in a flat position and gives the possibility of the joint to be self-deployed.

\section{Deployable CORE (D-CORE)}

\subsection{Description}

The deployable CORE (D-CORE) is made of two flat panels with curved creases that form the cams of the joint when deployed and three flexible bands which hold the two panels together. These components are chained together alternating between panels and flexible bands as is shown in Figures 5 and 6 . The two flat panels that form the cams also require curved cut edges if the angle between the cam sides and cam's contact surface varies from 90 degrees. The geometry of the curved cut edges and curved creases is discussed in Section 3.1.3. Two configurations of the D-CORE are presented below. Configuration 1 enables motion in the undeployed state while Configuration 2 does not. Both configurations can be manufactured from a single sheet, assembled by folding and one point of attachment, and are three layers thick in the assembled but undeployed state. Anchoring either configuration of D-COREs to rigid bodies can be achieved through sliding fits to accommodate for the motion of deployment. These sliding fits are discussed in Section 3.1.5 as an anchoring solution.

\subsubsection{Configuration 1}

Configuration 1 of the D-CORE enables a Jacob's ladder like motion when the joint is in its undeployed state and full CORE motion in the deployed state. It can be manufactured in a planar state (Figure 5(a)), folded into an assembled but undeployed flat state (Figure 5(b)), and deployed into the CORE geometry (Figure 5(c)). The length of the panels that form the cam surfaces, $L$, is equivalent to the length of the flexible bands, $L_{b a n d}$, when the bands are thin. The joint can be cut out of a single sheet or assembled from multiple components. If a single sheet is used for fabrication, one attachment point is required to complete a chain where the single flexible band passes between the two parallel flexible bands.

\subsubsection{Configuration 2}

The second configuration of the D-CORE is a static structure in its undeployed state and shows full CORE motion in its deployed state. Similar to the Configuration 1, Configuration 2 can be manufactured in a planar state (Figure $6(\mathrm{a})$ ), folded into an assembled but undeployed flat state (Figure 6(b)), and deployed into the CORE geometry (Figure 6(c)). Configuration 2 is constrained from motion in the undeployed state by placing hard stops on the panel's edges. One pair of these hard stops on each panel can be folded over themselves to accommodate for the thickness of the bands. This causes the panels to interfere with one another if the joint tries to rotate in the undeployed state. The addition of these hard stops slightly decreases the range of motion of the joint in the deployed position. 


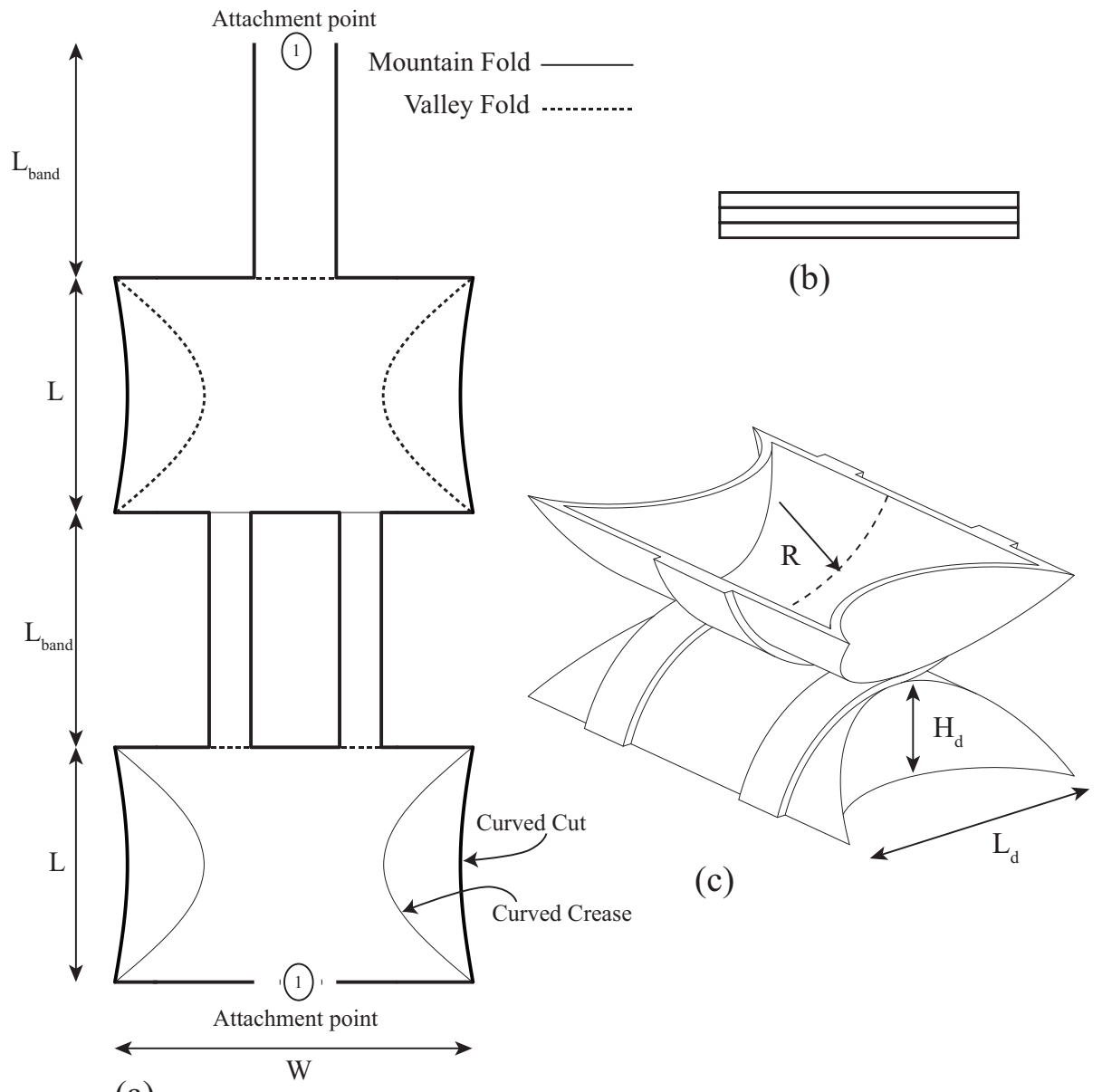

(a)

Figure 5: Configuration 1 of the D-CORE shown in its (a) planar, as-manufactured state, (b) folded undeployed state, and (c) deployed state. 


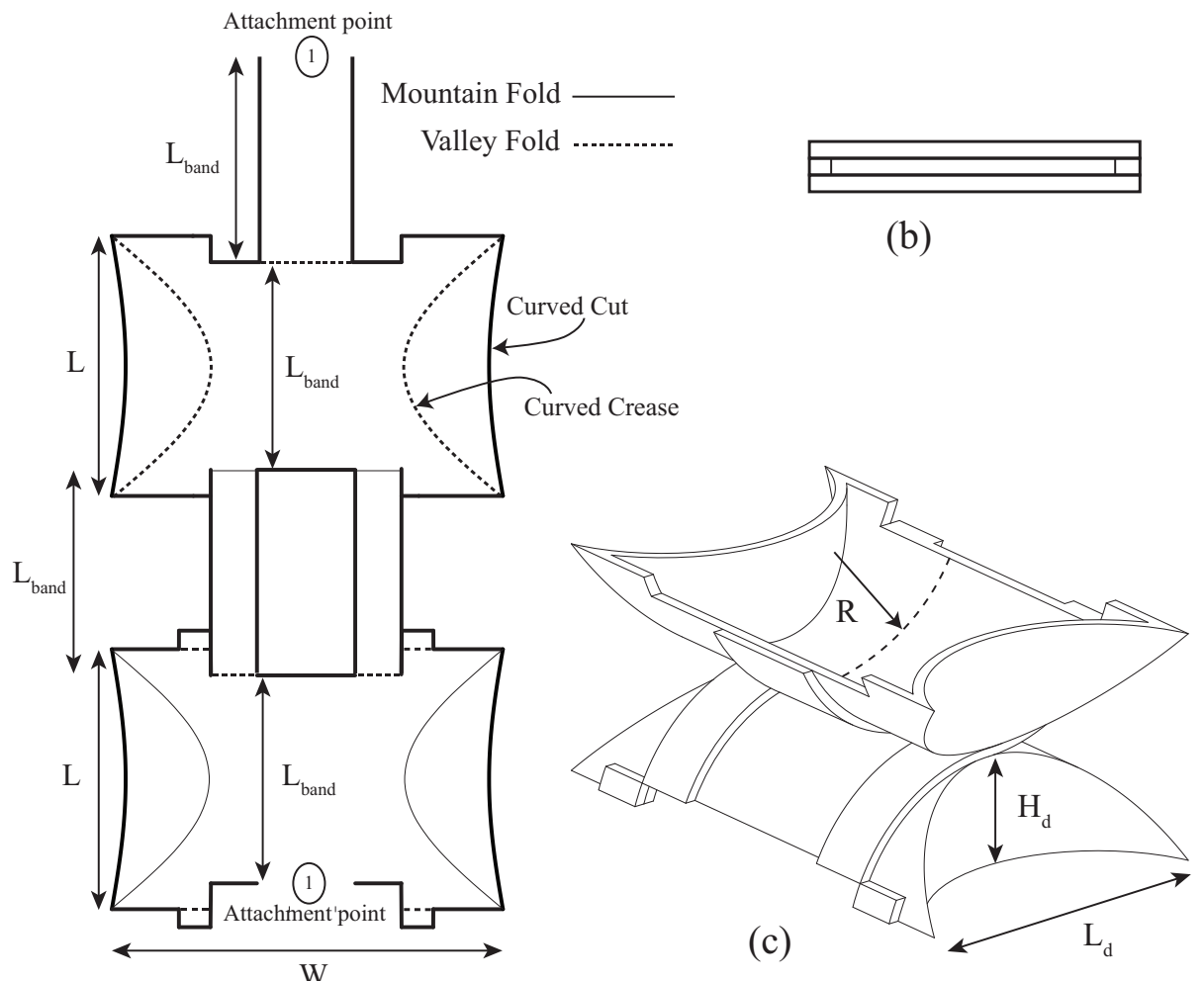

(a)

Figure 6: Configuration 2 of the D-CORE shown in its (a) planar as-manufactured state, (b) folded undeployed state, and (c) deployed state. 


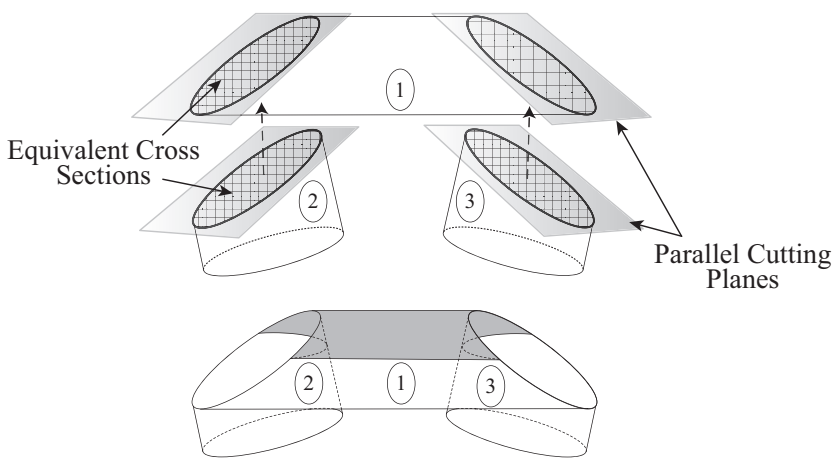

Figure 7: Modeling the CORE cam shown in gray as a combination of three oblique cylindrical sections intersected with one another.

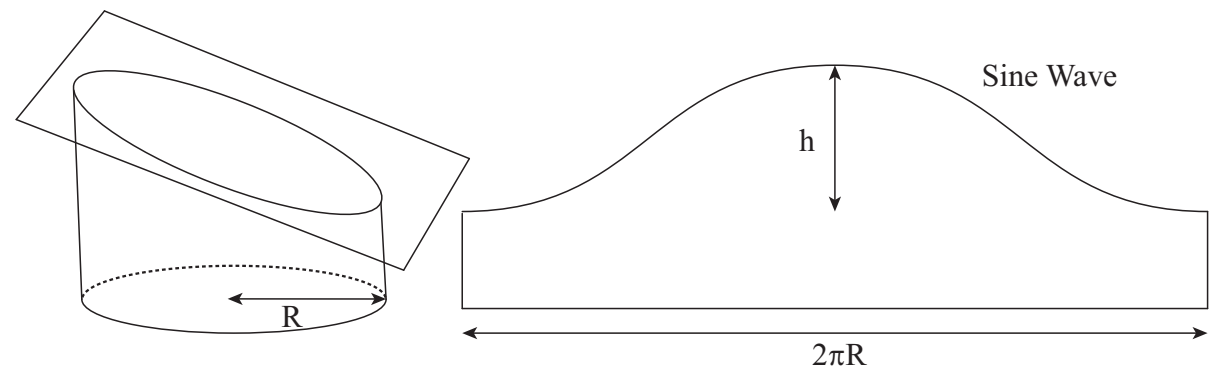

Figure 8: Intersection geometry for a cylinder and a cutting plane with the resulting unwrapped cylinder.

\subsubsection{Cam Construction Using Curved Creases}

Configurations 1 and 2 both draw upon curved creases to create the cam surfaces. The geometry of the curved creases used to form the cams as well as the length of the flexible bands of the D-CORE determines the range of motion for the angular deflection of the joint and the radius of the cam. The crease geometry can be determined by considering the intersection of cylinders where the cam face is modeled as a section of a cylinder and the two cam side panels are also modeled as sections of cylinders, as shown in Figure 7. Apostol et al. [27] show that the intersection curve between a cylinder and a plane is an elliptical cross section and when the cut cylinder is unwrapped the profile of the cut edge is a sine wave, as shown in Figure 8. To form a deployable CORE cam, the cutting planes of three cylinders are placed on top of one another and the elliptical cross sections are aligned as shown in Figure 7 . When any cylinder is unwrapped the profile of the cut edge forms the geometry of the curved crease. If the angle between the face and sides of the deployed cam, $\alpha$, differs from $\pi / 2$ the panels that form the cams will need to have an additional curved cut edge to enable the joint to sit on a flat plane in the deployed state.

The equations describing the relationship between the range of angular de-

Preprint: Published article available with DOI 
Deployed Cam

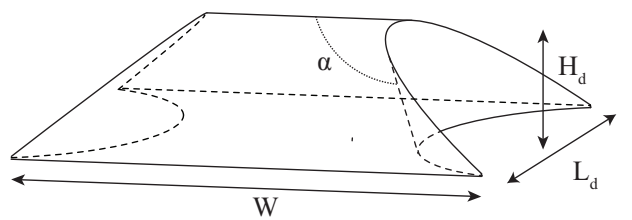

Undeployed Cam

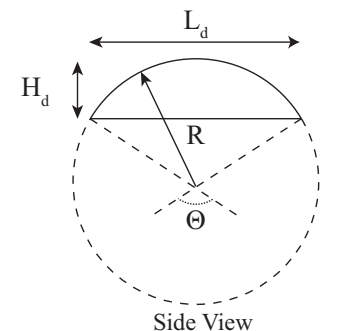

Side View

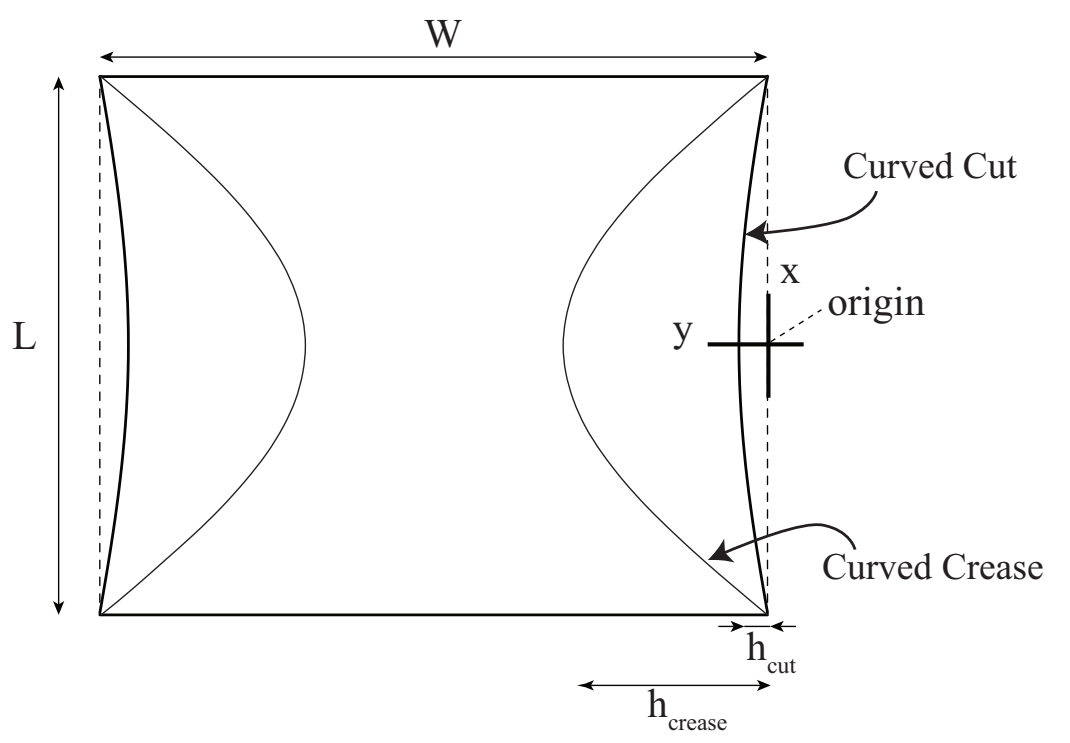

Figure 9: Parameters for a cylindrical cam with cylindrical side panels in the undeployed and deployed states.

flection of the joint in radians, $\Theta$, the radius of a cylindrical cam, $R$, the angle between the face and sides of the deployed cam in radians, $\alpha$, and the geometry of the curved crease and curved cuts expressed as functions, $y_{\text {crease }}(x)$ and $y_{\text {cut }}(x)$, can be determined for deployable joints with cylindrical side panels of the cams as is described in the next section. Figure 9 shows the parameters which will be used in the expressions.

\subsubsection{Deployable CORE Model for Design}

The following relationships were developed for the D-CORE such that the radius of the cam, $R$, the deflection of the joint, $\Theta$, the angle between the cam sides and face, $\alpha$, and the width of the cam, $W$, are selected and all other parameters of the D-CORE are computed. After selecting the radius of the cylindrical cam, $R$, and the desired angular deflection of the joint, $\Theta$, which can 
range from 0 to $\pi$, the required panel length, $L$, can be calculated by

$$
L=R \Theta
$$

It should be noted that the addition of the hard stops in Configuration 2 causes the effective angular deflection of the joint to be decreased to

$$
\Theta_{\text {config } 2}=\frac{L_{b a n d}}{R}
$$

Using the selected angle in radians between the cam face and side panels in the deployed state, $\alpha$, the height of the crease, $h_{\text {crease }}$, is computed as follows

$$
h_{\text {crease }}=R \tan \left(\frac{\alpha}{2}\right)\left(1-\cos \frac{\Theta}{2}\right)
$$

The height of the curved cut edge of the panel, $h_{c u t}$, when $\alpha$ is in radians is described as

$$
h_{c u t}=R \tan \left(\alpha-\frac{\pi}{2}\right)\left(1-\cos \frac{\Theta}{2}\right)
$$

The crease geometry, which corresponds to the unwrapped curve of a cylinder cut by a plane, can then be expressed by the following equation with the coordinate system placed as shown in Figure 9.

$$
y_{\text {crease }}(x)=R \tan \left(\frac{\alpha}{2}\right)\left(\cos \frac{x}{R}-\cos \frac{\Theta}{2}\right)
$$

where the domain for $x$ is from $-\frac{L}{2}$ to $\frac{L}{2}$. This expression was derived in a manner similar to that described by Apostol et al. [27] for unwrapping curves from cylinders. Similarly, the curved cut edge geometry, which also corresponds to the unwrapped curve of a cylinder, can be expressed by the following equation where the coordinate system is placed as shown in Figure 9

$$
y_{\text {cut }}(x)=R \tan \left(\alpha-\frac{\pi}{2}\right)\left(\cos \frac{x}{R}-\cos \frac{\Theta}{2}\right)
$$

The length of the deployed cam, $L_{d}$, is expressed as

$$
L_{d}=2 R \sin \frac{\Theta}{2}
$$

The height of the deployed cam, $H_{d}$, is computed from

$$
H_{d}=R\left(1-\cos \frac{\Theta}{2}\right)
$$

The length of the bands are shown in Figures 5 and 6 for the two configurations.

While the D-CORE cams created with these equations are circular cylindrical cams in the final deployed state, the surfaces are not guaranteed to remain as circular cylinders during the deployment of the cam from a flat state. The 
shape the surface of the cam takes during the deployment process can be investigated using the process outlined by Apostol et al. [27] of finding the shape of a generalized cylinder cut by a plane where the unwrapped curve of intersection is known. The generalized cylindrical shape can be considered as the profile of the cam while the plane shown in Figure 7 can be considered the cutting plane. The unwrapped curve of intersection is the cam's crease geometry as described in Equation 5. This crease geometry is constant for any stage of the deployment process.

\subsubsection{Anchoring the D-CORE to Rigid Bodies}

The joint boundaries move during deployment which complicates anchoring the D-CORE to rigid bodies. One method of anchoring the joint while still allowing for the deployment motion is to use sliding fits as shown in Figure 10. These fits are joined to the cam panels with a crease. The length of the sliding fit is $\left(L-L_{d}\right) / 2$ to accommodate the deployment motion. If the anchor points can be placed underneath the deployed cam, the range of motion of the joint will not be affected, yet the undeployed folded state will be an additional two layers thick. If the anchor points are placed on the outside of the cam, the range of motion will be decreased due to interference of the anchors at the extreme positions of the joint. Also, if the angle between the sides of the cam and the cam's contact surface, $\alpha$, falls between the range of 0 to $3 \pi / 4$, interference can occur during deployment as the sides of the cam swing below the anchoring plane. To ensure clearance, appropriate cuts can be made in the panels to which the D-CORE is anchored.

\subsubsection{Stiffness and Stress Characteristics of the D-CORE}

The D-CORE manufactured from a single sheet is unlikely to have the same performance in compression as a solid CORE. However, the deployability of the D-CORE gives it advantages besides compressive strength which can make the joint suitable to applications requiring a small stowed volume or low-cost manufacturing. The development of a simple analytical model for stiffness and stress of the D-CORE under various loading conditions is challenging because of the combined effects of stress from deployment and the stress induced by the joint loading. As such, prototype testing and finite element analysis methods are recommended for determining the stiffness and stress characteristics for particular applications of the D-CORE.

Despite the difficulty in developing a simple analytical model, insight can be gained by comparing finite element models of hollow CORE and D-CORE cams with the same dimensions, material, and compressive loads. Two finite element models (ANSYS, BEAM 188) were created to represent hollow CORE and D-CORE cams. The hollow CORE cam was modeled as an initially curved beam fixed at both ends as shown in Figure 11. The cam of the D-CORE was modeled as an initially flat beam which was morphed into its deployed shape using moment loads on either end of the beam. As the curved creases of the DCORE cam force the initially flat center panel to take the same circular shape as the moment loading, this method to create the deployed stresses was considered

Preprint: Published article available with DOI 


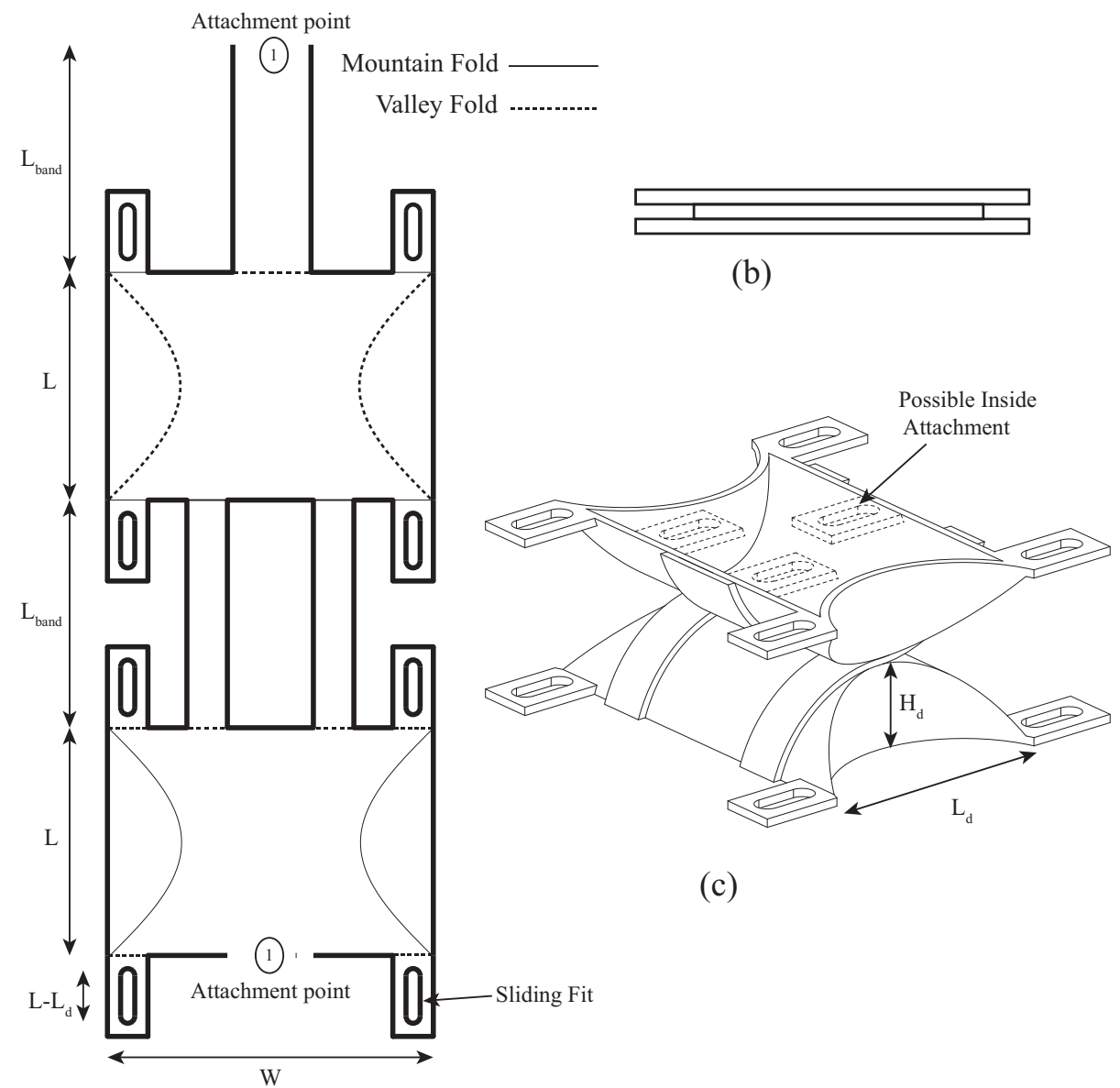

(a)

Figure 10: Anchoring the D-CORE using sliding fits shown in a position for (a) planar manufacture, (b) a folded undeployed state, and (c) a deployed state.

Preprint: Published article available with DOI 


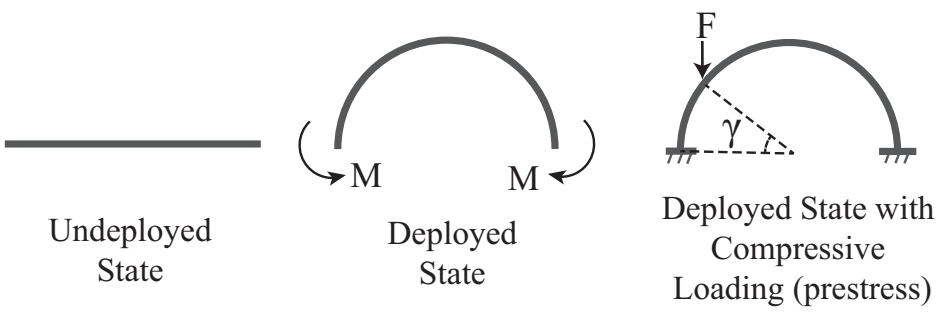

(a)

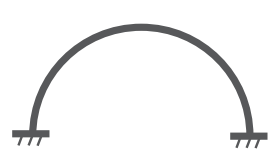

Unloaded State

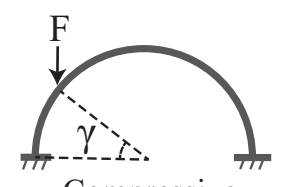

Compressive

Loading (no prestress)

(b)

Figure 11: Finite element model set up for (a) the D-CORE and (b) hollow CORE cams illustrating the presence of a prestress condition from deployment of the D-CORE and definition of the compressive load location parameter, $\gamma$.

as a reasonable approximation. Once in the deployed shape the ends of the beam were fixed and compressive loadings were applied at various locations around the cam as described by the load location parameter $\gamma$. The variation in the load location parameter was used to simulate the variation in the compressive loading location as the joint rolls through its range of motion.

These two finite element models were stress converged to within a $5 \%$ change in maximum Von Mises stress for two loading locations. The maximum Von Mises stress developed under the compressive loads at various locations is shown in Figure 12 for both the hollow CORE and D-CORE cams. The prestress component from the deployment of the D-CORE cam is reflected in the higher stresses present in the D-CORE cam for all of the loading locations. The deflection of the point where the compressive loading was applied was also plotted for both the hollow CORE and D-CORE cams in Figure 13. Interestingly the D-CORE cam shows a slight improvement in the stiffness at this point over the hollow CORE cam. While these finite element models give insight to general characteristics of the D-CORE performance under compressive loadings compared to a hollow CORE, further work would need to be conducted for specific applications. Also, analysis of the local stresses developed in the curved creases of the D-CORE rather than the curved panels during the loaded states needs to be explored.

\subsection{Physical Models}

Physical models of Configurations 1 and 2 were constructed out of a range of materials to demonstrate the transition from paper to engineering materials. 


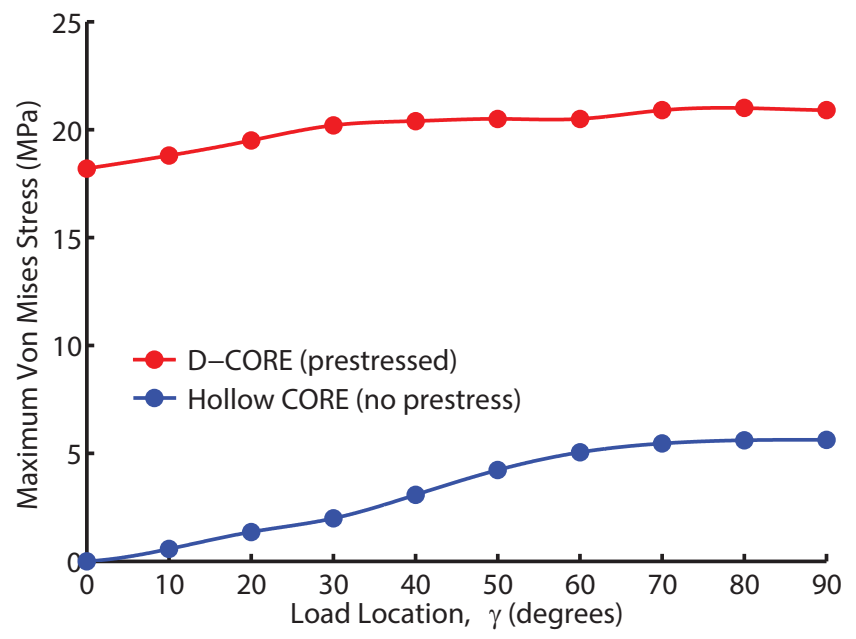

Figure 12: Maximum Von Mises Stress developed under compressive loadings at various locations for the hollow CORE and D-CORE cams.

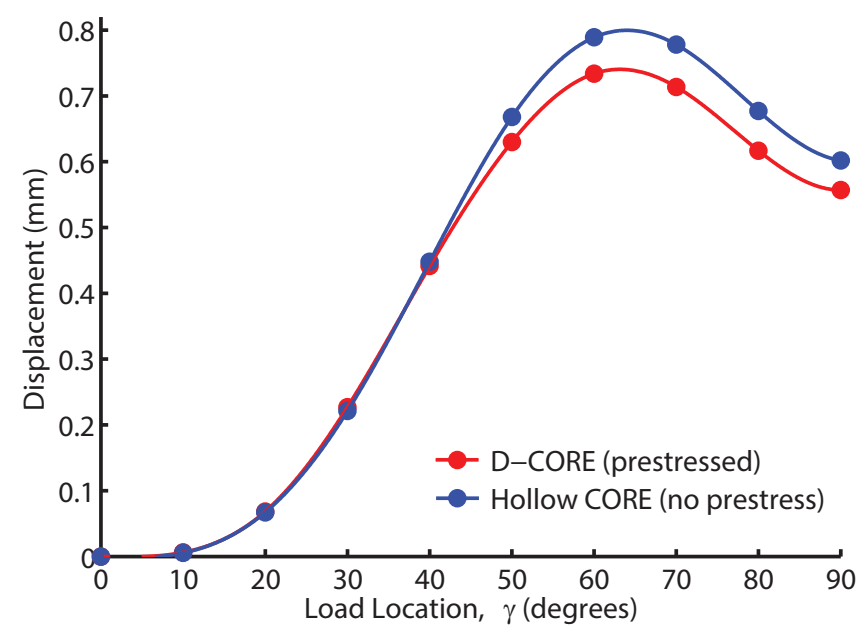

Figure 13: Displacement of the point where the compressive loading is applied for various loading locations for the hollow CORE and D-CORE cams.

Preprint: Published article available with DOI 


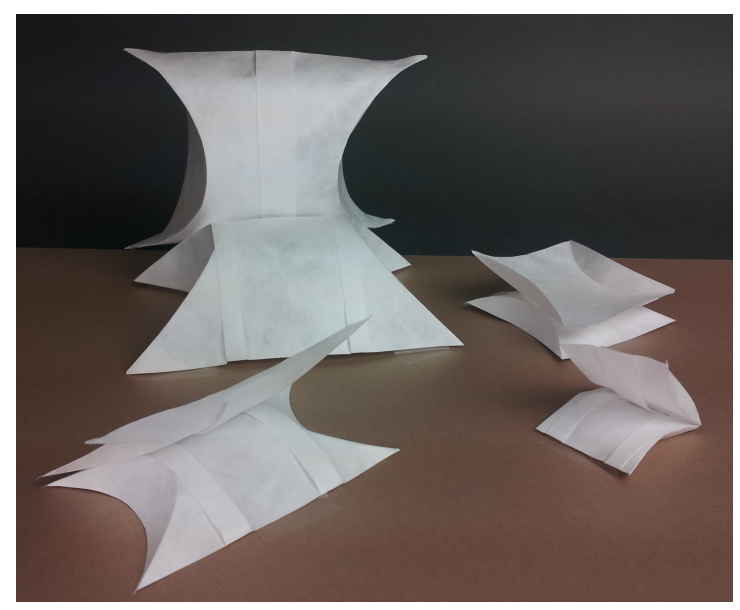

Figure 14: Tyvek $($ models of Configuration 1 with varying values of $\alpha$ and $\Theta$ and a common radius, $R$, of $5 \mathrm{~cm}$.

Figure 14 shows a family of Tyvek (B) models with common radius of the cylindrical cam, $R$, but varying values of the angle between the sides and contact surface of the cam, $\alpha$, and range of motion, $\Theta$. Tyvek $\AA$ is made from fusing together finely spun high density polyethylene fibers into a web. This results in a paper-like material that is tough, water resistant, and yet still maintains the ability to hold creases [28].

Configuration 1 was also constructed out of $0.38 \mathrm{~mm}(0.015 \mathrm{in})$ thick polycarbonate panels with $0.023 \mathrm{~mm}$ (9 mils) thick metallic glass hinges as shown in Figure 15. Bulk metallic glasses are metallic alloys which fail to crystallize during solidification. This gives them a unique combination of properties including high elastic strain and strength, making them a good candidate for compliant applications [29]. The metallic glass was secured with adhesive transfer tape between two layers of polycarbonate panels from which material in the curved crease regions had been removed. The joint was anchored to the foamboard panels by polycarbonate tabs that were inserted into slits in the foamboard.

A $0.81 \mathrm{~mm}$ (0.032 in) polypropylene sheet was used to construct Configuration 2 of the D-CORE. The polypropylene sheet was cut as shown in Figure 16(a). The curved creases and creases where the bands and sliding fits connect to the cam panels were created by using an embossing tool to remove material to form living hinges in the polypropylene. The polypropylene was folded and secured to wooden panels with nails to complete the sliding fits. Figure 16(b) and 16(c) show the D-CORE in the undeployed and deployed state. Polypropylene tabs on the base of the cams were inserted into grooves in the wood panels to lock the joint into the deployed state. The deployed joint functioned under a $100 \mathrm{~N}(22.5 \mathrm{lb})$ compressive load before visually detectable deformation of the cams occurred. 


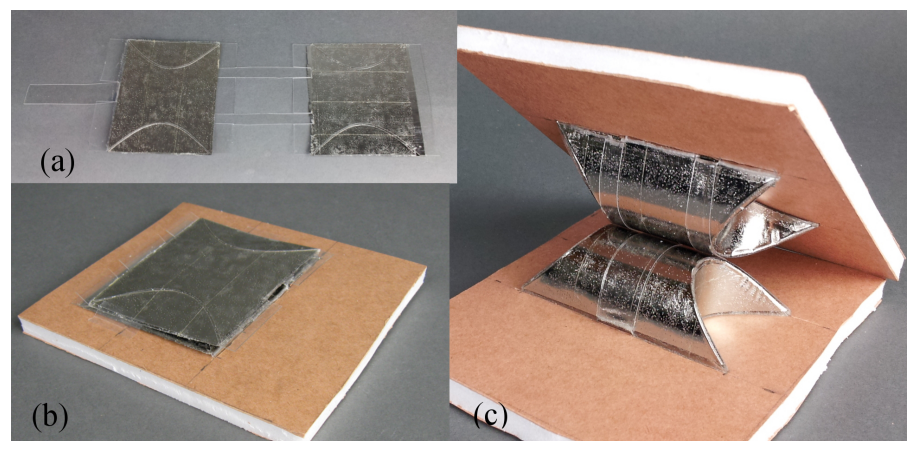

Figure 15: Configuration 1 made of polycarbonate panels and metallic glass hinges shown (a) during planar manufacture, (b) in a folded undeployed state without the top panel, and (c) in a deployed state

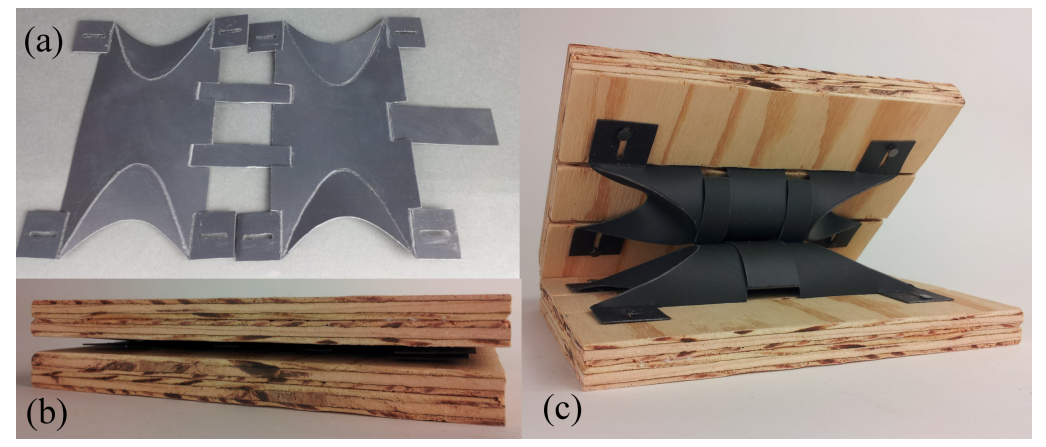

Figure 16: Configuration 2 made of polypropylene anchored to wood panels shown (a) during planar manufacture, (b) in a folded undeployed state, and (c) in a deployed state. 

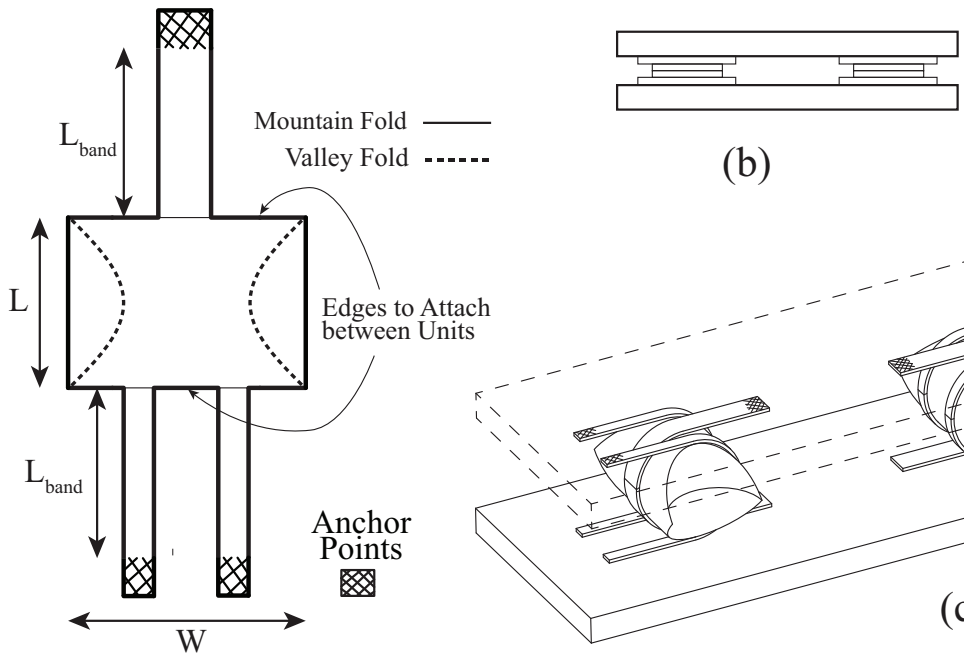

(b)

(a)

Figure 17: Translating platform geometry shown in (a) a planar state for manufacturing, (b) the assembled but undeployed state, and (c) the functioning deployed state.

\section{Deployable Translating Platform}

\subsection{Description}

By using two or more inverted D-CORE joints and adjusting the cam profile to be a half circle to enable 180 degrees of angular rotation, a deployable translating platform can be formed. The deployable translating platform has the ability to be stored flat with effectively no degrees of freedom and to deploy to a state where linear translation is facilitated. This change in the degrees of freedom between the stored and deployed state qualifies the translating platform as a metamorphic mechanism [2].

Figure 17 shows the geometry of the translating platform. Each of the joint units can be made from two identical pieces that are placed on top of one another and joined along the opposite edges of the panels which form the cams, as shown in Figure 17(a). These units are attached to the platforms by the flexible bands. The undeployed state is four layers thick (see Figure 17(b)). The deployed state of the platform is shown in Figure 17(c).

The range of linear translation, $\delta$, can be expressed in terms of the radius of the cam cylinder, $R$, as

$$
\delta=\pi R
$$

This range can be easily adjusted to a shorter length by shortening the length of the flexible bands. The cam geometry and curved crease geometry are described by Equations (1),(3),(5),(7), and (8) where $\Theta=\pi$. If $\alpha<3 \pi / 4$, the two cam units joined together will need to be actuated at separate times to avoid

Preprint: Published article available with DOI 


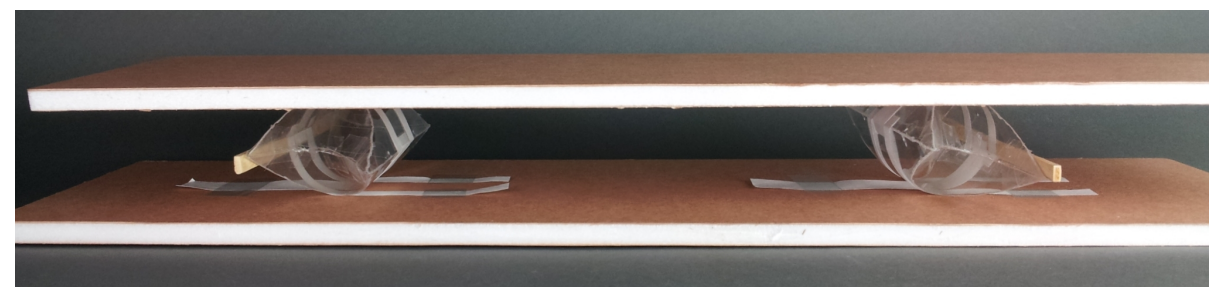

Figure 18: Translating platform constructed of polycarbonate cams attached to translating panels via Tyvek@ flexible bands.

interference of the cam side panels. If $\alpha \geq 3 \pi / 4$ the cams can be actuated simultaneously and furthermore all edges of the cam units can be joined together instead of only the opposite edges shown in Figure 17(a). The motion that occurs during deployment of the translating platform does not require sliding fits to anchor the joint units to the translating panels because the joint units can be anchored to the translating panels by the flexible bands.

\subsection{Physical Model}

A model of the translating platform was constructed from deployable cams made of $0.38 \mathrm{~mm}$ (0.015 in.) polycarbonate sheets and connected to foamboard translating panels via Tyvek® flexible bands. A small piece of wood and mono-filament lines were used to lock the mechanism into the deployed position. Figure 18 shows the physical model of the translating platform in the deployed state.

\section{Discussion}

As the D-CORE can be stowed in a flat configuration it can be used in applications where space constraints would limit the use of a traditional CORE. These applications include minimally invasive medical implants, space applications, and stowable furniture. However, when the cams of the CORE are made to be deployable, the compressive strength of the joint decreases.

Actuation of the joint can be accomplished through a force which pulls the edges of the deployable cams together or through forces which fold the creases to the appropriate angles. Springs or hand actuation, as was used with the models in this work, are two methods of deploying the joint by forcing the edges of the deployable cam together. Shape memory alloys and thermally actuated contractile polymers $[30,31]$ also present possible methods of self-folding or deployment of the joints. Inflation is a possible actuation method for the cams of the translating platform if $\alpha \geq 3 \pi / 4$ and all edges of the cam units are joined together. 


\section{Conclusion}

This work applied curved-fold origami techniques to create the geometry of a D-CORE that is manufacturable from a single sheet. Two configurations were presented which have the ability to be stored in a flat state and deploy to a functioning state, making them suitable for space-constrained applications such as medical devices and in aerospace. The crease geometry was mathematically related to key properties of the D-CORE to facilitate design of the joint. These relations were used to create physical prototypes of the D-CORE. A deployable translating platform was presented as a derivative of the D-CORE.

This research outlines how the geometry of the D-CORE is created for a cylindrical radius. It may be possible to characterize other profiles which can be created using curved creases. These non-cylindrical profiles could be used to

create multiple stable positions of the joint and also to change the location of the instantaneous axis of rotation.

\section{Acknowledgments}

This material is based on work supported by the National Science Foundation and the Air Force Office of Scientific Research under NSF Grant EFRIODISSEI-1240417.

\section{References}

[1] P. A. Halverson, L. L. Howell, S. P. Magleby, Tension-based multi-stable compliant rolling-contact elements, Mechanism and Machine Theory 45 (2) (2010) $147-156$.

[2] J. S. Dai, J. R. Jones, Mobility in metamorphic mechanisms of foldable/erectable kinds, Journal of Mechanical Design 121 (3) (1999) 375-382.

[3] Jacob's ladder, Scientific American October 12, 1889 Issue.

[4] X. Ding, Y. Yang, J. S. Dai, Topology and kinematic analysis of colorchanging ball, Mechanism and Machine Theory 46 (1) (2011) 67-81.

[5] L. L. Howell, Compliant mechanisms, John Wiley \& Sons, 2001.

[6] D. F. Wilkes, Rolamite: A new mechanical design concept, unclassified No. SC-RR-67-656, Sandia Corporation (1967).

[7] R. V. Cadman, Rolamite: geometry and force analysis, Tech. rep., Sandia Corporation (1970).

[8] A. Thornton, P. Predecki, Design considerations in a rolamite knee joint prosthesis, Journal of Biomedical Materials Research 7 (3) (1973) 419-433.

Preprint: Published article available with DOI 
[9] C. English, D. Russell, Implementation of variable joint stiffness through antagonistic actuation using rolamite springs, Mechanism and Machine Theory 34 (1) (1999) 27-40.

[10] P. A. Halverson, A. E. Bowden, L. L. Howell, A compliant-mechanism approach to achieving specific quality of motion in a lumbar total disc replacement, The International Journal of Spine Surgery 6 (1) (2012) 7886.

[11] T. Y. Nai, J. L. Herder, G. J. Tuijthof, Steerable mechanical joint for high load transmission in minimally invasive instruments, Journal of Medical Devices 5 (3) (2011) 034503.

[12] Y.-M. Moon, Bio-mimetic design of finger mechanism with contact aided compliant mechanism, Mechanism and Machine Theory 42 (5) (2007) 600611.

[13] Z. You, Folding structures out of flat materials, Science 345 (6197) (2014) 623-624.

[14] G. Wei, J. S. Dai, Origami-inspired integrated planar-spherical overconstrained mechanisms, Journal of Mechanical Design 136 (5) (2014) 051003.

[15] Y. Qin, J. S. Dai, G. Gogu, Multi-furcation in a derivative queer-square mechanism, Mechanism and Machine Theory 81 (2014) 36-53.

[16] C. Qiu, V. Aminzadeh, J. S. Dai, Kinematic analysis and stiffness validation of origami cartons, Journal of Mechanical Design 135 (11) (2013) 111004.

[17] K. Zhang, J. S. Dai, A kirigami-inspired 8r linkage and its evolved overconstrained $6 \mathrm{r}$ linkages with the rotational symmetry of order two, Journal of Mechanisms and Robotics 6 (2) (2014) 021007.

[18] D. Fuchs, S. Tabachnikov, More on paperfolding, American Mathematical Monthly (1999) 27-35.

[19] D. J. Struik, Lectures on classical differential geometry, 2nd Edition, Reading, Mass., Addison-Wesley Pub. Co., 1961.

[20] H. Pottmann, A. Schiftner, P. Bo, H. Schmiedhofer, W. Wang, N. Baldassini, J. Wallner, Freeform surfaces from single curved panels, ACM Transactions on Graphics (TOG) 27 (3) (2008) 76.

[21] V. Ushakov, Developable surfaces in euclidean space, Journal of the Australian Mathematical Society (Series A) 66 (03) (1999) 388-402.

[22] D. A. Huffman, Curvature and creases: A primer on paper, IEEE Trans. Computers 25 (10) (1976) 1010-1019.

Preprint: Published article available with DOI 
[23] R. D. Koschitz, Computational design with curved creases : David huffman's approach to paperfolding, Ph.D. thesis, Massachusetts Institute of Technology (2014).

[24] R. D. Resch, Portfolio of shaded computer images, Proceedings of the IEEE 62 (4) (1974) 496-502.

[25] R. J. Lang, One ellipse to rule them all, The Fold (Origami USA), 2013.

[26] E. D. Demaine, M. L. Demaine, D. Koschitz, T. Tachi, Curved crease folding: a review on art, design and mathematics, in: Proceedings of the IABSE-IASS Symposium, 2011.

[27] T. M. Apostol, M. A. Mnatsakanian, Unwrapping curves from cylinders and cones, American Mathematical Monthly 114 (5) (2007) 388-416.

[28] K. Francis, J. Blanch, S. Magleby, L. Howell, Origami-like creases in sheet materials for compliant mechanism design, Mechanical Sciences 4 (2) (2013) $371-380$.

[29] E. R. Homer, M. B. Harris, S. A. Zirbel, J. A. Kolodziejska, H. Kozachkov, B. P. Trease, J.-P. C. Borgonia, G. S. Agnes, L. L. Howell, D. C. Hofmann, New methods for developing and manufacturing compliant mechanisms utilizing bulk metallic glass, Advanced Engineering Materials 16 (7) (2014) 850-856.

[30] M. T. Tolley, S. M. Felton, S. Miyashita, D. Aukes, D. Rus, R. J. Wood, Self-folding origami: shape memory composites activated by uniform heating, Smart Materials and Structures 23 (9) (2014) 094006.

[31] S. Felton, M. Tolley, E. Demaine, D. Rus, R. Wood, A method for building self-folding machines, Science 345 (6197) (2014) 644-646.

Preprint: Published article available with DOI 